NYHA II) but absence of pathological findings in electrocardiogram (ECG), echocardiography and lung function were investigated by CMR. CMR was conducted with a 1.5 Tesla MRI-System (Achieva, Philips, Best, Netherlands) using a 32channel coil. T2 mapping was done using a respiration navigator gated Gradient and Spin-Echo sequence (GRASE, 15 T2 echoes separated by $10 \mathrm{~ms}$, res: $1 \times 1 \times 10 \mathrm{~mm}^{2}, 3$ short axis slices). Images were post-processed using software based on the LabView environment for local T2 value generation (T2 mapping). Strain analysis was conducted entering cineimages into myocardial feature tracking (FTI) analysis software (TomTec Imaging Systems, Unterschleißheim, Germany). A cohort eleven of age and gender matched healthy controls (HC) served as controls.

Results All SLE patients showed significantly extended T2 times as a sign of local inflammation compared with age matched healthy controls $(\mathrm{p}<0.05)$. Moreover, the global systolic longitudinal strain (GLS) as means by systolic function was significantly decreased. In addition, global early diastolic strain rate displayed diastolic dysfunction in comparison to controls.

Conclusions SLE patients with persistent dyspnoea in absence of pathological findings in ECG and echocardiography showed significantly extended T2-times in MRI as a sign of local fluid content as a part of myocardial inflammation, reduced GLS and diastolic dysfunction, which would be missed by using conventional technics. CMR and T2-mapping is a possible tool for the investigation of a cardiac involvement in SLE patients and should be investigated in clinical studies.
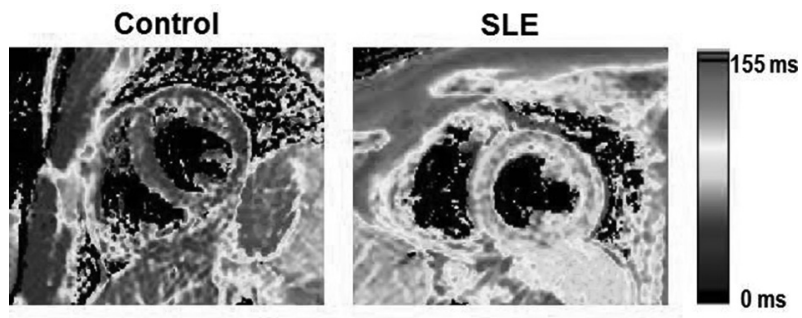

Abstract PS6:123 Figure 1

\section{PS6:124 ALGORITHMS TO IDENTIFY SLE FROM EHR DATA}

R Ramsey-Goldman, T Walunas, K Jackson, A Chung, D Erickson, K Mancera-Cuevas, A Kho. Northwestern Univerity Feinberg School of Medicine, Chicago, USA

\subsection{6/lupus-2018-abstract.167}

Background SLE is difficult to diagnose because of the diverse manifestations occurring over time and across care sites. Electronic health records (EHR) present a rich source of patient information which can be mined for diagnosis and identification to improve quality of care or to enrol patients in studies. Aim Build a rule-based algorithm for each revised 1982/1997 ACR classification criteria for SLE using EHR data.

Methods We included patients from the Chicago Lupus Database (CLD) fulfilling 4 or more of the ACR classification criteria for SLE who also had records in the Northwestern Medicine Electronic Data Warehouse (NMEDW) EHR. ICD-9 codes and lab test results for each ACR SLE criterion were ascertained. We queried patient diagnoses, lab results and used a simple chart string for lab test results from physician notes.
Abstract PS6:124 Table 1 Comparing the frequency of received ACR classification criteria for CLE identified in two database, CLD (disease specific) and NMEDW (EHR)

\begin{tabular}{|c|c|c|}
\hline & CLD & NMEDW \\
\hline Malar/Discoid Rash & $41.8 \% / 6.7 \%$ & $42.3 \% / 0 \%$ \\
\hline Photosensitivity/Oral Ulcers & $\begin{array}{c}62.3 \% / 42.6 \\
\%\end{array}$ & $7 \% / 17.6 \%$ \\
\hline Arthritis/Serositis & $\begin{array}{c}82.9 \% / 39.7 \\
\%\end{array}$ & $5.8 \% / 48.6 \%$ \\
\hline Renal/Neurologic Disorder & $39.7 \% / 7.7 \%$ & $50.7 \% / 17.1 \%$ \\
\hline Immunologic Disorder & $71.9 \%$ & $27.9 \%$ \\
\hline Anti-Nuclear Antibody & $95 \%$ & $24 \%$ \\
\hline Hematologic Disorder & $54.8 \%$ & $23.6 \%$ \\
\hline
\end{tabular}

Results Data from 515/783 patients in CLD and the NMEDW EHR were included. When using ICD 9 codes only $8.8 \%$ of patients from CLD/NMEDW were identified. With the addition of lab results to the query concordance increased to $54.7 \%$, and a simple text string query to search physician notes for additional lab results increased identification to $57.5 \%$.

Conclusion Using ICD codes plus laboratory data from NMEDW increased fulfilment of classification criteria but is still not optimal for patient identification. Additional strategies such as using natural language processing (NLP) or examining fulfilment of SLICC classification criteria for SLE which includes more lab results than ACR may yield an improved rule-based algorithm for the identification of SLE patients in EHR data.

\section{PS6:125 SYSTEMIC LUPUS ERYTHEMATOSUS DIAGNOSIS IS EARLIER IN MALES COMPARED TO FEMALES}

E Gozcu, A Karatas, B Oz, SS Koca. Department of Rheumatology, Faculty of Medicine, Firat University, Elazig, Turkey

\subsection{6/lupus-2018-abstract.168}

Background Systemic lupus erythematosus (SLE) is a chronic autoimmune disease. It affects both men and women, with a female predominance. On the other hand, many different studies have showed that disease manifestations and their severities are different in the males and females. The aim of our study was to detect whether sex variation leads to diagnostic delay.

Methods SLE patients, those admitted our outpatient clinic between January 2017 and August 2017, were enrolled in the study. 46 patients fulfilling the SLICC classification criteria for SLE and regularly followed at our rheumatology clinic were selected. At the time of enrollment, medical and pharmacological histories were collected. Patients, those have $>6$ months duration between starting symptoms to diagnosis, were accepted to have diagnostic delay. Statistical comparisons were made using Mann Whitney $U$ test and chi-square tests.

Results Diagnostic delay was detected in the $25 \%$ of males and $73.8 \%$ of females $(p=0.043)$. The diagnosis was earlier in the males compared to the females (OR: $2.7,95 \%$ CI: 1.3 to 6.1 ).

Conclusion SLE is a multisystem complex autoimmune disease that often mimics symptoms of other illnesses. Many SLE manifestations such as fatigue, hair loss, arthralgia and anaemia in especially females are confounding for clinicians in the diagnostic process. Clinicians carefully investigate the diagnosis for these manifestations in males, since various frequent diseases are suspected in females. Therefore, SLE diagnosis is earlier in males compared to females. 
Abstract PS6:125 Table 1 Data in male and female SLE patients

\begin{tabular}{|l|c|c|c|}
\hline & Males $(\mathbf{n = 4})$ & Females $(\mathbf{n = 4 2})$ & $\mathbf{P}$ \\
\hline Age, years & $41.5 \pm 12.8$ & $33.6 \pm 9.5$ & 0.308 \\
\hline Symptom duration, years & $5.5 \pm 6.4$ & $6.4 \pm 5.6$ & 0.763 \\
\hline Disease duration, years & $5.3 \pm 6.5$ & $5.5 \pm 5.3$ & 0.950 \\
\hline Hemoglobin, g/dl & $12.3 \pm 2.9$ & $11.6 \pm 1.8$ & 0.509 \\
\hline Platelet, 10 $3 / \boldsymbol{\mu l}$ & $339.8 \pm 138.9$ & $252.8 \pm 109.8$ & 0.145 \\
\hline WBC, $\mathbf{1 0}$ / $\boldsymbol{\mu l}$ & $4.4 \pm 2.6$ & $7.5 \pm 12.1$ & 0.190 \\
\hline ESR, $\mathbf{~ m m / h}$ & $34.5 \pm 33.5$ & $39.6 \pm 28.9$ & 0.743 \\
\hline CRP, $\mathbf{~ m g / d l ~}$ & $2.1 \pm 1.6$ & $0.6 \pm 1.2$ & 0.025 \\
\hline ANA positive, \% & 75 & 88.1 & 0.457 \\
\hline
\end{tabular}

WBC: White blood cell count, ESR: erythrocyte sedimentation rate, $C R P$ : C-reactive protein, $A N A$ : anti-nuclear antibody

\section{REFERENCES}

1. Oglesby A, et al. Impact of early versus late systemic lupus erythematosus diagnosis on clinical and economic outcomes. Appl Health Econ Health Policy 2014;12:179-90.

2. Boodhoo KD, et al. Impact of sex disparities on the clinical manifestations in patients with systemic lupus erythematosus: A systematic review and meta-analysis. Medicine (Baltimore) 2016;95:e4272.

\section{PS6:126 AUTOIMMUNE CYTOPENIA IN SYSTEMIC LUPUS ERYTHEMATOSUS: EXPERIENCE OF AN INTERNAL MEDIC}

G Chalhoub. Central Regional Hospital of Mercy, Ars Laquenexy, France

\subsection{6/lupus-2018-abstract.169}

Objective Our objective was to review the rate of autoimmune cytopenia observed in lupus patients either at the inaugural diagnosis of lupus or during an already known lupus disease; try to correlate it with the precipitating factors and evaluate their outcome.

Design and Method This is a retrospective study of 9 years in an adult unit of internal medicine including 108 lupus adult patients. Anti-nuclear antibodies (ANA) were present in all patients, with antibodies to double stranded DNA (anti dsDNA) \pm anti nucleosome antibodies.

It concerns 94 women and 14 men. 35 cases diagnosed after the age of 50 .

Results The autoimmune cytopenia was observed in 21 patients (16 women and 5 men).

The revelation modes varied between: exclusive neutropenia (6 cases), exclusive thrombocytopenia(6 patients)and pancytopenia ( 9 patients).

The association with antiphospholipid antibodies was noted in 6 patients, 2 cases had in addition insignificant titre of cold agglutinin, 1 case with a Sjögren syndrom, 2 cases associated with rheumatoid arthritis, 1 case with sclerosing cholangitis, 1 patient with cryofibrinogenemia and 2 cases with multiple autoimmunity disease. Only 1 woman had vitamin B12 deficiency.
The entry into the disease was revealed by a thrombotic microangiopathy (TMA) in one case and another woman by HELLP syndrom. A bone marrow aspirate was performed in a number of patients and eliminated a central cause.

Treatment with HYDROXYCHLOROQUINE and corticosteroids was given to all patients.

Immunosuppressive therapy, intravenous Immunoglobulin, thrombopoetin agonists and anti-CD20 were used in some cases. Cyclic antibiotics and Granocyte-colony stimulating factor were given to a patient with profound neutropenia and repeated severe infections. Plasma exchanges were performed in the TMA patient. The outcome was generally favourable for most patients and did not present a risk for survival. The death occurred in 2 patients due to other causes (mesenteric ischemia in one and bladder cancer in another).

Conclusions The autoimmune cytopenia in lupus is a source of diagnostic wandering, especially when it inaugurates the clinical presentation. The best knowledge of the mechanisms makes it possible to adapt the appropriate treatment. These cytopenias in our series did not constitute a pejorative element and were always of good prognosis due to the early management.

\section{PS6:127 GENDER INFLUENCE IN SYSTEMIC LUPUS ERYTHEMATOSUS}

M Kechida, R Mesfar, R Klii, S Hammami, I Khochtali. Internal Medicine and Endocrinology Department, Monastir, Tunisia

\subsection{6/lupus-2018-abstract.170}

Introduction Systemic lupus erythematosus (SLE) is more frequent in men than women with sex ratio $\mathrm{F} / \mathrm{M}=8 / 1$, but whether it's more severe or not, is not clear

Objectives We aimed to study clinical, biological and immunological features of SLE in men.

Methods It's a retrospective study conducted in an internal medicine department. Patients with systemic lupus erythematosus (ACR revised criteria) were included. Data were recorded 